\title{
An Underwater Imaging System Layout Method based on Minimum Illumination and Contrast Calculation
}

\author{
Guixin Sun ${ }^{1, a}$ \\ The People's Liberation Army Unit 91550, Dalian, 116023, China \\ aemail: GuixinSDLI@163.com
}

Keywords: Underwater Imaging; Minimum Illumination; Minimum Contrast

\begin{abstract}
Underwater imaging is an effective method to measure the moving objects in water. Based on the attenuation characteristics of light in water, the underwater imaging system layout method based on the calculation of minimum illumination and minimum contrast is proposed in this paper. This method as underwater imaging system technical conditions, combined with underwater environment, auxiliary light source design, underwater light field layout, seeking to gain a clear high quality underwater image system layout solution, provide technical support for the construction of underwater imaging system.
\end{abstract}

\section{Introduction}

Underwater imaging system is under dim illumination and highly scattering media conditions in sea water, on the water environment in the event of clear imaging equipment. In the marine environment, the technical condition of the underwater imaging system is related to the characteristics of underwater target, the condition of the water medium, the technical performance of the imaging system and the motion performance of the installation platform.

Working in the marine environment of the imaging system, due to the use of the environment, the use of conditions and other restrictions, and the theoretical design of the role of distance there is a certain gap. Due to the influence of the attenuation of light in water, light is absorbed and scattered by the water body, the absorption makes the photon disappear, and the energy is converted into other forms of energy [1]. The scattering makes the traveling direction of photons change, and the radiation intensity decreases. The mechanism of light attenuation in sea water is due to the effect of sea water on the absorption and scattering of light [2]. The attenuation of light in the sea water is exponentially decaying with the light attenuation coefficient. On the one hand, due to the absorption of the underwater light field energy in the continuous decline, but the absorption effect does not affect the light in the transmission process of the formation of optical field distribution; on the other hand, due to the scattering function of photon to change direction, forming a new underwater light field distribution and for the diffuse light field [3] [4]. Under the water, the image in the water transfer process is not only the perspective of transmission, but also the brightness, contrast transfer [5].

Through the calculation of minimum illumination and contrast, this paper improves the analysis method of underwater imaging distance, and provides some guidance and reference for the actual imaging measurement.

\section{Technical Conditions of Underwater Imaging System}

The working process of underwater target imaging to receiver is influenced by many factors. The light transmission characteristics in water make the underwater imaging system work under the condition of low illumination, which requires the underwater imaging system has high sensitivity and high resolution. Minimum imaging requirements for underwater imaging systems: target image is above two pixels on the detector; the illumination value of the target is higher than the lowest sensitivity value in the detector; the contrast ratio of the target to the detector's target is more than $0.2[6]$. 
The working schematic diagram of underwater imaging system is shown in Figure 1. It can be seen from the figure that the key to the imaging of a certain distance is the illumination of the object. That is the object of the water in the water, after the water transfer to the imaging system, the object side of the intensity of the imaging system to meet the minimum requirements of imaging.

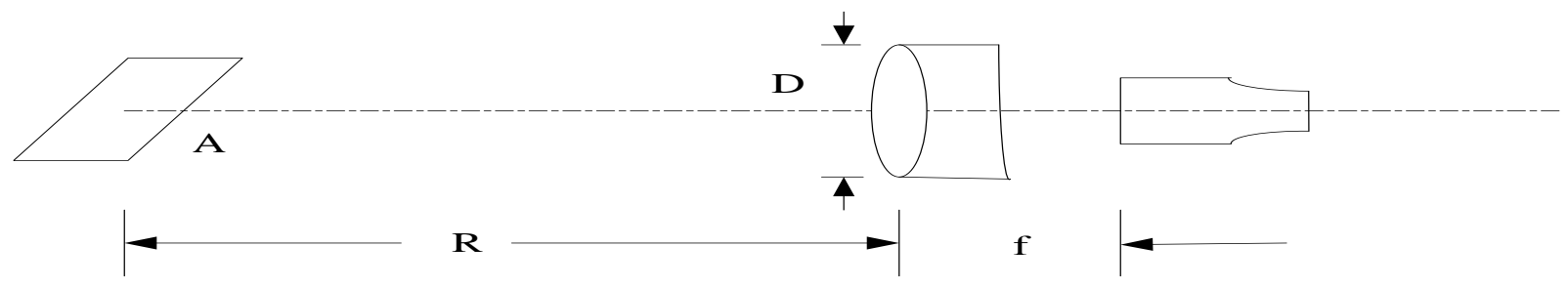

Fig.1. Operation flow chart for the underwater imaging system

$R$ is the imaging system's function distance; $D$ is the effective aperture of the optical system; $F$ is the focal length of the optical system; $A$ is the measured target.

The measured object can be seen as a Lambertian radiator, attenuation according to the exponential decay rule in illumination. Underwater image intensity transfer process of natural light through the sea, a certain depth of sea water transfer attenuation to reach the target, the target was measured after the absorption and scattering of sea water to reach the camera image surface.

The minimum illumination of the target under water is:

$$
E_{p i x}=\frac{\rho \tau E_{o b j}}{4 F^{2}} e^{-c R}
$$

$E_{p i x}$ is the illumination of the image received by the receiver; $E_{o b j}$ is the illumination of object; $F$ is aperture value; $P$ is target surface reflectivity; $\tau$ is the transmittance of optical system; $c$ is transmission attenuation coefficient for different wavelengths; $R$ is the function distance of imaging system.

The decrease of contrast of underwater imaging is mainly due to the natural light in the water is very diffuse, and cannot be projected out of the shadow. The brightness contrast between the underwater target and the surrounding background will gradually become smaller after passing through the water. The contrast can be expressed by the following formula:

$$
C_{0}=\left|\left(B_{m}-B_{b}\right) / B_{b}\right|
$$

$C_{0}$ is contrast between target and background, $B_{\mathrm{m}}$ is Underwater target brightness, $B_{\mathrm{b}}$ is brightness of underwater background. According to the definition, combined with the process of underwater image transmission analysis, can be sorted out as follows:

$$
\left\{\begin{array}{c}
C_{R}=C_{0} e^{-\alpha R} \\
C_{0}=\left|\beta e^{-\alpha R}-1\right| \\
\beta=\left(\rho \tau e^{-\alpha R}\right) / 4 F^{2}
\end{array}\right.
$$

\section{Experiment and Results}

To detect the water at a certain distance from the target, must meet three conditions: target at the receiver energy is higher than the sensitivity threshold; target and background contrast at the receiver is greater than the receiver threshold; target at the receiver line imaging is greater than the resolution limit. From the process of energy transfer, the sensitivity is the signal current generated by the unit optical power. The effective signal voltage, which is obtained by the unit exposure, reflects the minimum radiation power or the minimum illumination intensity that can be sensed by the device. The target's contrast ratio is the difference between the target and the radiation intensity of the surface of the target. 
By the formula (3), the numerical values of the variation of the image surface light intensity with water depth and distance are calculated as shown in Table 1 . The variation trends of image surface illumination with water depth and distance are shown in Figure 2 and Figure 3.

Tab.1. Comparison of imaging luminance with depth and distance changing (unit:1/x)

\begin{tabular}{|c|c|c|c|}
\hline \multirow{2}{*}{ Depth(z)m } & \multicolumn{3}{|c|}{$E_{R}$} \\
\cline { 2 - 4 } & $R_{1}=5 \mathrm{~m}$ & $R_{2}=10 \mathrm{~m}$ & $R_{3}=15 \mathrm{~m}$ \\
\hline 10 & 3.857 & 1.854 & 0.891 \\
\hline 15 & 1.803 & 0.867 & 0.417 \\
\hline 20 & 0.844 & 0.406 & 0.195 \\
\hline 25 & 0.407 & 0.195 & 0.094 \\
\hline 30 & 0.198 & 0.095 & 0.046 \\
\hline 40 & 0.052 & 0.025 & 0.012 \\
\hline
\end{tabular}

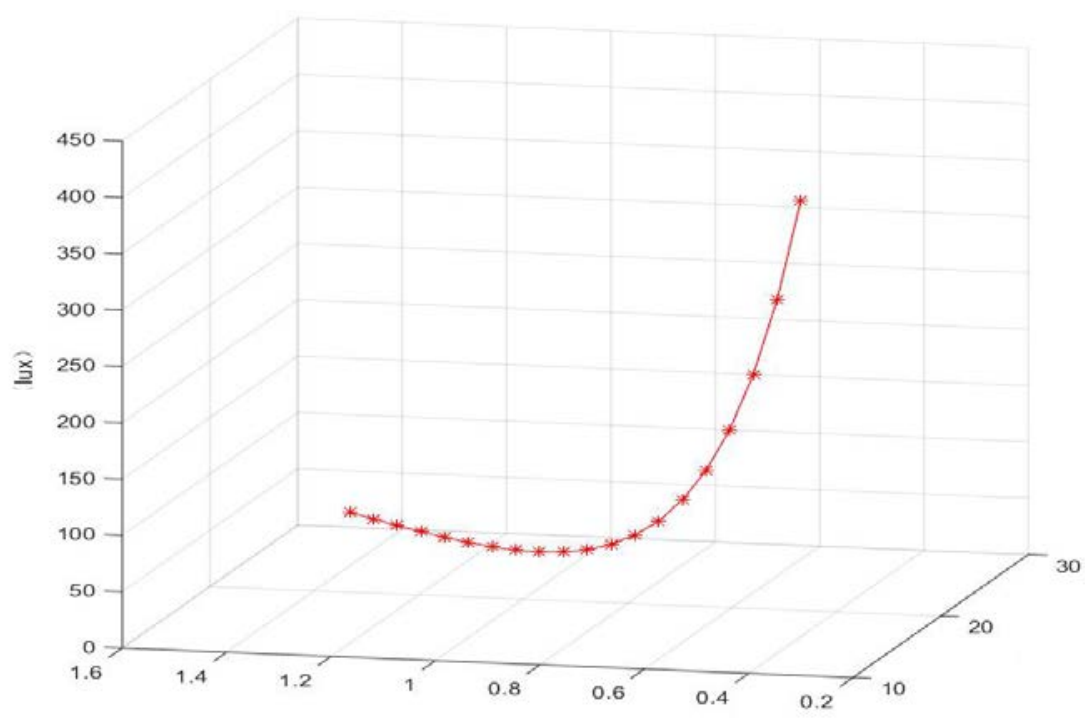

Fig.2. The experimental results

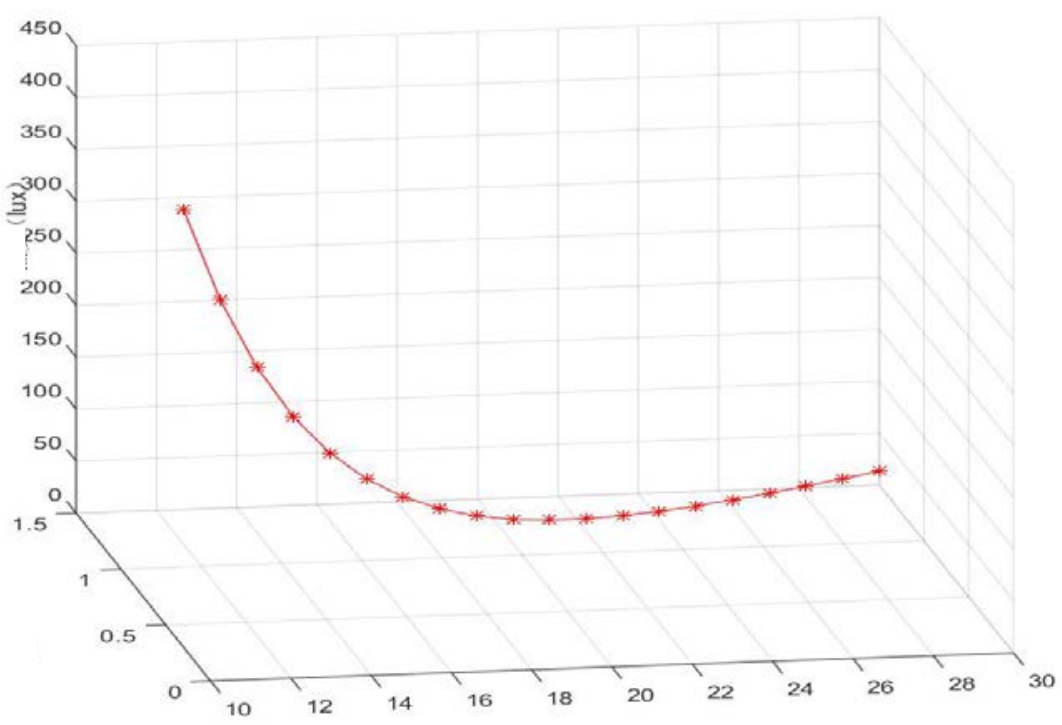

Fig.3. The experimental results 
Under certain conditions, the optical transmittance and relative aperture of the imaging system can be determined by the illumination of the object, the action distance and the size of the sea water attenuation coefficient. In the surface of the material, the attenuation coefficient of the sea water is small, and the intensity of the image surface is larger. When the light energy into the camera is larger than the minimum sensitivity of the camera, the minimum contrast of the image is not related to the depth of the water. Under certain conditions, the influence of the sea water attenuation coefficient is larger. The transparency of sea water is large, and the image surface is large, which is good for underwater imaging.

\section{Conclusion}

Based on the energy and contrast of the image information, the mathematical expression of the illumination and contrast of the image output is deduced, which can be used as a system to detect the target. The technical performance of underwater imaging system is designed according to the task of carrying on. Influenced by target characteristic, underwater environment characteristic, working platform and camera's own technical performance. Underwater imaging system design to system analysis and calculation, to underwater imaging technology as the foundation, give full consideration to the characteristics of target, the water under the premise of complex application environments, scientific design of auxiliary illuminant, reasonable layout of auxiliary light source position, selected to satisfy the use requirements of the imaging system. The proposed method can provide technical support for the measurement of the underwater moving target.

\section{References}

[1] HE Zhao-cai, Hu Bao-n. The Light Measurement System M].Beijing: National Defense Industry Press, 2002, 1.

[2] XIANG Shi-ming, NI Guang-qiang, et al. The Principle of Photo electronic Imaging Devices [M].Beijing: National Defense Industry Press, 1999, 4.

[3] WU Zheng, SUN Ao, et al. Test and Measurement control Technique for Sea Range [M]. Beijing: National Defense Industry Press, 2007, 12.

[4] Xu Hong-mei. Study on target detection of underwater inhomogeneous light field [D]. Qingdao: Ocean University of China Press, 2009.

[5] Li Yan. Performance evaluation of underwater low light level imaging system based on energy transfer chain [J]. Journal of missile and guidance, 2010, 5(196-198).

[6] Sun Chuan-dong. Optical properties of water and its effect on underwater imaging [J]. Applied Optics, 2007, 21(4):39-46. 